\title{
Precompact Sets, Boundedness, and Compactness of Commutators for Singular Integrals in Variable Morrey Spaces
}

\author{
Wei Wang ${ }^{1}$ and Jingshi $\mathrm{Xu}^{2}$ \\ ${ }^{1}$ School of Sciences, Central South University of Forestry and Technology, Changsha 410004, China \\ ${ }^{2}$ Department of Mathematics, Hainan Normal University, Haikou 571158, China \\ Correspondence should be addressed to Jingshi Xu; jingshixu@126.com
}

Received 10 May 2017; Accepted 18 June 2017; Published 20 July 2017

Academic Editor: Dashan Fan

Copyright ( 2017 Wei Wang and Jingshi Xu. This is an open access article distributed under the Creative Commons Attribution License, which permits unrestricted use, distribution, and reproduction in any medium, provided the original work is properly cited.

We give sufficient conditions for subsets to be precompact sets in variable Morrey spaces. Then we obtain the boundedness of the commutator generated by a singular integral operator and a BMO function on the variable Morrey spaces. Finally, we discuss the compactness of the commutator generated by a singular integral operator and a BMO function on the variable Morrey spaces.

\section{Introduction}

Let the Calderón-Zygmund singular integral operator $T$ be defined by

$$
T f(x):=\text { p.v. } \int_{\mathbb{R}^{n}} \frac{\Omega(x-y)}{|x-y|^{n}} f(y) \mathrm{d} y, \quad \forall x \in \mathbb{R}^{n},
$$

where $\Omega$ is a measurable function on $\mathbb{R}^{n}$ and satisfies the following conditions:

(i) $\Omega$ is a homogeneous function of degree zero on $\mathbb{R}^{n} \backslash$ $\{0\}$; that is,

$$
\Omega(\mu x)=\Omega(x) \text { for any } \mu>0, x \in \mathbb{R}^{n} \backslash\{0\} .
$$

(ii) $\Omega$ has mean zero on $S^{n-1}$; that is,

$$
\int_{S^{n-1}} \Omega\left(x^{\prime}\right) \mathrm{d} \sigma\left(x^{\prime}\right)=0 .
$$

Here $S^{n-1}:=\left\{x \in \mathbb{R}^{n}:|x|=1\right\}$ is the unit sphere in $\mathbb{R}^{n}$ and $\mathrm{d} \sigma$ is the area measure on it.

For a function $b \in L_{\mathrm{loc}}\left(\mathbb{R}^{n}\right)$ (the set of all locally integrable functions on $\mathbb{R}^{n}$ ), let $M_{b}$ be the corresponding multiplication operator defined by $M_{b} f=b f$ for a measurable function $f$. Then the commutator between $T$ and $M_{b}$ is denoted by

$$
\begin{aligned}
{[b, T] } & :=M_{b} T-T M_{b} \\
& =\text { p.v. } \int_{\mathbb{R}^{n}} \frac{\Omega(x-y)}{|x-y|^{n}}(b(x)-b(y)) f(y) \mathrm{d} y,
\end{aligned}
$$

for suitable functions $f$. Denote the bounded mean oscillation function space by

$$
\begin{aligned}
& \operatorname{BMO}\left(\mathbb{R}^{n}\right)
\end{aligned}
$$

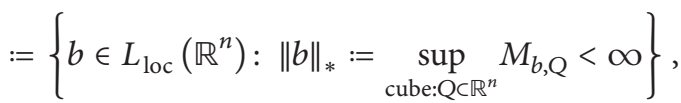

here and in the sequel

$$
\begin{aligned}
& M_{b, Q}:=\frac{1}{|Q|} \int_{Q}\left|b(x)-b_{\mathrm{Q}}\right| \mathrm{d} x, \\
& b_{\mathrm{Q}}:=\frac{1}{|Q|} \int_{\mathrm{Q}} b(y) \mathrm{d} y .
\end{aligned}
$$

It is well known that commutators play a very important role in harmonic analysis and PDEs. Indeed, Coifman et al. [1] characterized the $L^{p}$-boundedness of $\left[b, R_{j}\right]$, where 
$R_{j}(j=1, \ldots, n)$ are the Reisz transforms and $b \in \operatorname{BMO}\left(\mathbb{R}^{n}\right)$. Using this characterization, the authors of [1] obtained a decomposition theorem of the real Hardy spaces $H^{1}\left(\mathbb{R}^{n}\right)$. Uchiyama [2] and Janson [3] showed that the Riesz transform $R_{j}$ may be replaced by the Calderón-Zygmund singular integral operator $T$ as in (1). Coifman et al. generalized the boundedness results of $[b, T]$ to Hardy spaces and gave important applications to some nonlinear PDEs in [4]. The characterization of $L^{p}$-compactness of $[b, T]$ was obtained by Uchiyama [2]. We remark that the interest in the compactness of $[b, T]$ in complex analysis is from the connection between the commutators and the Hankel-type operators; see [5]. In recent years, Chen et al. have considered the compactness of commutators in [6-8]. Specially, the results in [2] were generalized to Morrey spaces in [8]. The Morrey space $L^{p, \lambda}\left(\mathbb{R}^{n}\right)$ was introduced by Morrey in 1938 and it is connected to certain problems in elliptic PDE [9]. After that the Morrey spaces were found to have many important applications to the Navier-Stokes equations (see [10]), the Schrödinger equations (see [11]), and potential theory (see [12-14]).

During last three decades, the theory of variable function spaces has developed quickly; see [15-40]. We claim that the list is not exhaust. The boundedness in variable function spaces of many classical operators from harmonic analysis has been obtained; see [18, 19, 41-44]. Motivated by these works, we will consider analogous results in [8] to variable exponent situation. The structure of this paper is as follows. In Section 2, we give sufficient conditions for a set to be a precompact set in a variable Morrey space. In Section 3, we obtain the boundedness of singular integrals and their commutator in variable Morrey spaces. In Section 4, we discuss compactness of commutators in variable Morrey spaces. The remainder of this section is some notions.

Let $E$ be a measurable subset in $\mathbb{R}^{n}$ with $|E|>0$, where as usual $|E|$ is the Lebesgue measure of $E$. Let $p(\cdot)$ be a measurable function on $E$ with range in $[1, \infty)$. The variable exponent modulus is defined for measurable functions $f$ on E by

$$
\rho_{p(\cdot)}(f)=\int_{E}|f(x)|^{p(x)} \mathrm{d} x<\infty .
$$

$L^{p(\cdot)}(E)$ denotes the set of measurable functions $f$ on $E$ such that $\rho_{p(\cdot)}(\lambda f)<\infty$ for some $\lambda>0$. The set becomes a Banach function space when equipped with the norm

$$
\|f\|_{L^{p(\cdot)}(\Omega)}:=\inf \left\{\lambda>0: \rho_{p(\cdot)}\left(\frac{f}{\lambda}\right) \leq 1\right\} .
$$

These spaces are the so-called variable Lebesgue spaces. Denote by $\mathscr{P}^{1}(E)$ the set of measurable functions $p(\cdot)$ on $E$ with range in $[1, \infty)$ such that

$$
\begin{aligned}
1<p^{-} & :=\text {ess } \inf _{x \in E} p(x), \\
\text { ess } \sup _{x \in E} p(x) & =: p^{+}<\infty .
\end{aligned}
$$

For $p(\cdot) \in \mathscr{P}^{1}\left(\mathbb{R}^{n}\right)$ and $0<\lambda(x)<n$ for $x \in \mathbb{R}^{n}$, the variable Morrey space $L^{p(\cdot), \lambda(\cdot)}\left(\mathbb{R}^{n}\right)$ is defined as the set of integrable functions $f$ on $\mathbb{R}^{n}$ with the finite norm

$$
\|f\|_{L^{p(\cdot), \lambda(\cdot)}\left(\mathbb{R}^{n}\right)}:=\sup _{x \in \mathbb{R}^{n}, r>0} \frac{1}{v_{1} r^{\lambda(x) / p(x)}}\left\|f \chi_{B(x, r)}\right\|_{L^{p(\cdot)}\left(\mathbb{R}^{n}\right)},
$$

where $B(x, r)$ denotes a ball centered at $x$ with radius $r$ and $v_{1}$ is the volume of the unit ball in $\mathbb{R}^{n}$.

\section{Precompact Sets in Variable Morrey Spaces}

In this section, we give a compactness criterion in variable Morrey spaces. We remark here that a compactness criterion for variable exponent Lebesgue spaces was given in [45].

Theorem 1. Let $p(\cdot) \in \mathscr{P}^{1}\left(\mathbb{R}^{n}\right)$ and $0<\lambda(x)<n$ for $x \in \mathbb{R}^{n}$. Suppose $\mathscr{W}$ is a subset in $L^{p(\cdot), \lambda(\cdot)}\left(\mathbb{R}^{n}\right)$ satisfying the following conditions:

(i) Norm boundedness uniformly is

$$
\sup _{f \in \mathscr{W}}\|f\|_{L^{p(\cdot), \lambda(\cdot)}}<\infty
$$

(ii) Translation continuity uniformly is

$$
\lim _{y \rightarrow 0}\|f(\cdot+y)-f(\cdot)\|_{L^{p(\cdot), \lambda(\cdot)}}=0 \quad \text { for any } f \in \mathscr{W} .
$$

(iii) Uniformly convergence at infinity is

$$
\lim _{\alpha \rightarrow \infty}\left\|f \chi_{E_{\alpha}}\right\|_{L^{p(\cdot), \lambda(\cdot)}}=0 \quad \text { for any } f \in \mathscr{W} \text {, }
$$

where $E_{\alpha}=\left\{x \in \mathbb{R}^{n}:|x|>\alpha\right\}$.

Then $\mathscr{W}$ is a precompact set in $L^{p(\cdot), \lambda(\cdot)}\left(\mathbb{R}^{n}\right)$.

To prove Theorem 1, we need the following two lemmas, which are well known; for example, see [46].

Lemma 2. A set $E$ is precompact in a Banach space $X$ if and only if it is totally bounded which means for every positive number $\epsilon$ there is a finite subset $N_{\epsilon}$ of points of $X$ such that

$$
E \subset \bigcup_{y \in N_{\epsilon}} B_{\epsilon}(y)
$$

where $B_{\epsilon}(y)$ denotes a ball centered at $y$ with radius $\epsilon$. The set $N_{\epsilon}$ is called an $\epsilon$-net of $E$.

Lemma 3 (the Ascoli-Arzela theorem). Let $E$ be a bounded domain in $\mathbb{R}^{n}$. A subset $F$ of $C(\bar{\Omega})$ is precompact in $C(\bar{\Omega})$ if the following two conditions hold:

(i) There exists a constant $M$ such that $|u(x)| \leqslant M$ holds for every $u \in F$ and $x \in E$.

(ii) For every $\epsilon>0$, there exists $\delta>0$ such that $\mid u(x)-$ $u(y) \mid<\epsilon$ for $u \in F, x, y \in E$, and $|x-y|<\delta$. 
Now there is a position to prove Theorem 1 .

Proof of Theorem 1. Let $h>0$, we denote the mean of $f$ on $B(x, h)$ by

$$
M_{h} f(x):=\frac{1}{v_{1} h^{n}} \int_{y \in B(x, h)} f(x+y) \mathrm{d} y, \quad \text { for } x \in \mathbb{R}^{n}
$$

For $f \in L^{p(\cdot), \lambda(\cdot)}\left(\mathbb{R}^{n}\right)$ and $x \in \mathbb{R}^{n}$

$$
\begin{aligned}
& M_{h} f(x)-f(x) \\
& \quad=\frac{1}{v_{1} h^{n}} \int_{y \in B(x, h)}[f(x+y)-f(x)] \mathrm{d} y .
\end{aligned}
$$

Then by Hölder's inequality and Fubini's Theorem, for any $t \in$ $\mathbb{R}^{n}$ and $r>0$,

$$
\begin{aligned}
\left\|M_{h} f-f\right\|_{L^{p(\cdot)}(B(t, r))} & \leqslant C \sup _{\|g\|_{L^{p^{\prime}(\cdot)(B(t, r))}} \leqslant 1} \int_{x \in B(t, r)} \int_{y \in B(x, h)} \frac{1}{v_{1} h^{n}}(f(x+y)-f(x)) \mathrm{d} y g(x) \mathrm{d} x \\
& =C \sup _{\|g\|_{L^{p^{\prime}(\cdot)(B(t, r))}} \leqslant 1} \int_{y \in B(x, h)} \frac{1}{v_{1} h^{n}} \int_{x \in B(t, r)}(f(x+y)-f(x)) g(x) \mathrm{d} x \mathrm{~d} y \\
& \leqslant C \sup _{|y| \leqslant h}\|f(\cdot+y)-f(\cdot)\|_{L^{p(\cdot)}(B(t, r))} .
\end{aligned}
$$

Thus,

$$
\left\|M_{h} f-f\right\|_{L^{p(\cdot), \lambda(\cdot)}} \leqslant C \sup _{|y| \leqslant h}\|f(\cdot+y)-f(\cdot)\|_{L^{p(\cdot), \lambda(\cdot)}} \cdot
$$

Therefore, to prove that $\mathscr{W}$ is a precompact set, we need only to prove that $M_{h} \mathscr{W}$ is precompact for small $h$. By Lemma 2, it suffices to show that $M_{h} \mathscr{W}$ has finite $\epsilon$-net for any $\epsilon>0$. To do so, firstly, by Lemma 3, we show that $M_{h} \mathscr{W}$ is precompact in $C\left(E_{\alpha}^{c}\right)$ for each $\alpha>0$, where $E_{\alpha}^{c}=\left\{x \in \mathbb{R}^{n}:|x| \leqslant \alpha\right\}$. For any $x \in \mathbb{R}^{n}$, by Hölder's inequality,

$$
\begin{aligned}
& \left|M_{h} f(x)\right| \leqslant \frac{1}{v_{1} h^{n}} \int_{|y| \leqslant h}|f(x+y)| \mathrm{d} y \\
& \leqslant C \frac{1}{v_{1} h^{n}}\|f\|_{L^{p(\cdot)}(B(x, h))}\|1\|_{L^{p^{\prime}(\cdot)}(B(x, h))} \\
& \leqslant C \frac{h^{\lambda(x) / p(x)}}{h^{n}} \frac{1}{v_{1} h^{\lambda(x) / p(x)}}\|f\|_{L^{p(\cdot)}(B(x, h))}\|1\|_{L^{p^{\prime}(\cdot)}(B(x, h))} \\
& \leqslant C \frac{h^{\lambda(x) / p(x)}}{h^{n}}\|1\|_{L^{p^{\prime}(\cdot)}(B(x, h))}\|f\|_{L^{p(\cdot), \lambda(\cdot)}} \\
& \leqslant C(h, \lambda(\cdot), p(\cdot))\|f\|_{L^{p(\cdot), \lambda(\cdot)}},
\end{aligned}
$$

since $\|1\|_{L^{p^{\prime}(\cdot)}(B(x, h))} \leqslant \max \left\{\left(v_{1} h\right)^{1 / p^{\prime-}},\left(v_{1} h\right)^{1 / p^{\prime+}}\right\}$, and $h^{\lambda(x) / p(x)} \leqslant \max \left\{1, h^{n / p^{-}}\right\}$. Therefore, $M_{h} \mathscr{W}$ are uniformly bounded functions by Condition (i). Then for $x_{1}, x_{2} \in \mathbb{R}^{n}$,

$$
\begin{aligned}
& \left|M_{h} f\left(x_{1}\right)-M_{h} f\left(x_{2}\right)\right| \leqslant \frac{1}{v_{1} h^{n}} \\
& \cdot \int_{|y| \leqslant h}\left|f\left(x_{1}+y\right)-f\left(x_{2}+y\right)\right| \mathrm{d} y \leqslant C \\
& \cdot \frac{1}{v_{1} h^{n}}\left\|f\left(x_{1}-x_{2}+\cdot\right)-f(\cdot)\right\|_{L^{p(\cdot)}\left(B\left(x_{2}, h\right)\right)}
\end{aligned}
$$

$$
\begin{aligned}
& \cdot\|1\|_{L^{p^{\prime}(\cdot)}(B(x, h))} \leqslant C(h, \lambda(\cdot), p(\cdot)) \\
& \cdot\left\|f\left(x_{1}-x_{2}+\cdot\right)-f(\cdot)\right\|_{L^{p(\cdot), \lambda(\cdot)}} .
\end{aligned}
$$

Thus, by Condition (ii) and Lemma $3, M_{h} \mathscr{W}$ is precompact in $C\left(E_{\alpha}^{c}\right)$.

Finally, we verify that $M_{h} \mathscr{W}$ has finite $2 \epsilon$-net for each small positive $\epsilon$. For $0<\epsilon<1$, there exist $N>0$ and $\alpha>0$ such that $1<\epsilon^{-N} / 4<\alpha^{n / p^{-}}<\epsilon^{-N} / 2$ and for each $f \in \mathscr{W}$

$$
\left\|f_{E_{\alpha}}\right\|_{L^{p(\cdot), \lambda(\cdot)}}<\frac{\epsilon}{8} \text {. }
$$

By Lemma 3, there exist $\left\{f_{1}, f_{2}, \ldots, f_{m}\right\} \subset \mathscr{W}$ such that $\left\{M_{h} f_{1}, M_{h} f_{2}, \ldots, M_{h} f_{m}\right\}$ is a finite $\epsilon^{N+1}$-net in $M_{h} \mathscr{W}$ in the norm of $C\left(E_{\alpha}^{c}\right)$. Below we verify that $\left\{M_{h} f_{1}, M_{h} f_{2}, \ldots\right.$, $\left.M_{h} f_{m}\right\}$ is a finite $\epsilon$-net in $M_{h} \mathscr{W}$ in the norm of $L^{p(\cdot), \lambda(\cdot)}$.

To finish the proof, we only need to show that, for $f \in \mathscr{W}$, there is a $f_{j}(j \in\{1,2, \ldots, m\})$ such that for $r>0, t \in \mathbb{R}^{n}$

$$
I:=\frac{1}{v_{1} r^{\lambda(t) / p(t)}}\left\|M_{h} f-M_{h} f_{j}\right\|_{L^{p(\cdot)}(B(t, r))}<\epsilon .
$$

Now, we choose $f_{j}, j \in\{1,2, \ldots, m\}$ such that

$$
\sup _{y \in E_{\alpha}^{c}}\left|M_{h} f(y)-M_{h} f_{j}(y)\right|<\epsilon^{N+1} .
$$

To show (22), we consider $B(t, r)$ into three cases.

Case 1. $B(t, r) \subset E_{\alpha}^{c}$.

If $r \leqslant 1$,

$$
I \leqslant \frac{1}{v_{1} r^{\lambda(t) / p(t)}} \epsilon^{N+1} r^{n / p^{+}} \leqslant \epsilon^{N+1} r^{(n-\lambda(t)) / p^{+}}<\epsilon .
$$

If $r>1$,

$$
I \leqslant\left\|M_{h} f-M_{h} f_{j}\right\|_{L^{p(\cdot)}\left(E_{\alpha}^{c}\right)} \leqslant \epsilon^{N+1} \alpha^{n / p^{-}}<\epsilon .
$$


Case 2. $B(t, r) \subset E_{\alpha}$. Thus, we have

$$
\begin{aligned}
I= & \frac{1}{v_{1} r^{\lambda(t) / p(t)}}\left\|\left(M_{h} f-M_{h} f_{j}\right) \chi_{E_{\alpha}}\right\|_{L^{p(\cdot)}(B(t, r))} \\
& \leqslant \frac{1}{v_{1} r^{\lambda(t) / p(t)}}\left(\left\|\left(M_{h} f-f\right) \chi_{E_{\alpha}}\right\|_{L^{p(\cdot)}(B(t, r))}\right. \\
& \left.+\left\|\left(f-f_{j}\right) \chi_{E_{\alpha}}\right\|_{L^{p(\cdot)}(B(t, r))}\right) \leqslant \| M_{h} f \\
& \left.+\left\|\left(f_{j}-M_{h} f_{j}\right) \chi_{E_{\alpha}}\right\|_{L^{p(\cdot)}(B(t, r))}\right)\|\| \chi_{E_{\alpha}}\left\|_{L^{p(\cdot), \lambda(\cdot)}}+\right\| f_{E_{\alpha}}\left\|_{L^{p(\cdot), \lambda(\cdot)}}+\right\| f_{j} \\
& -f\left\|_{L^{p(\cdot), \lambda(\cdot)}}+\right\| f . \\
& -M_{h} f_{j} \|_{L^{p(\cdot), \lambda(\cdot)}}<\epsilon .
\end{aligned}
$$

Case 3. $B(t, r) \cap E_{\alpha}^{c} \neq \emptyset$, and $B(t, r) \cap E_{\alpha} \neq \emptyset$. Hence,

$$
\begin{aligned}
I \leqslant & \frac{1}{v_{1} r^{\lambda(t) / p(t)}}\left\|\left(M_{h} f-M_{h} f_{j}\right) \chi_{E_{\alpha}}\right\|_{L^{p(\cdot)}(B(t, r))} \\
& +\frac{1}{v_{1} r^{\lambda(t) / p(t)}}\left\|\left(M_{h} f-M_{h} f_{j}\right) \chi_{E_{\alpha}^{c}}\right\|_{L^{p(\cdot)}(B(t, r))} \\
:= & I_{1}+I_{2} .
\end{aligned}
$$

Here, $I_{1}, I_{2}$ can be estimated that $I_{1}, I_{2}<\epsilon$ by Cases 1 and 2 , respectively.

Therefore, $M_{h} \mathscr{W}$ has a finite $2 \epsilon$-net in $L^{p(\cdot), \lambda(\cdot)}\left(\mathbb{R}^{n}\right)$. This completes the proof.

\section{Boundedness of Singular Integrals and Their Commutators}

To consider the boundedness of singular integrals, a fundamental operator is the Hardy-Littlewood maximal operator. Given a function $f$, the maximal function $M f$ is defined by

$$
M f(x)=\sup _{\mathrm{Q} \ni x} \frac{1}{|Q|} \int_{\mathrm{Q}}|f(y)| \mathrm{d} y, \quad \forall x \in \mathbb{R}^{n},
$$

where the supremum is taken over all cubes containing $x$. It is well known that $M$ is bounded on $L^{p}, 1<p<\infty$. However, for any $p(\cdot) \in \mathscr{P}^{1}\left(\mathbb{R}^{n}\right), M$ need not be bounded in $L^{p(\cdot)}\left(\mathbb{R}^{n}\right)$. Let $\mathscr{B}\left(\mathbb{R}^{n}\right)$ be the set of $p(\cdot) \in \mathscr{P}^{1}\left(\mathbb{R}^{n}\right)$ such that $M$ is bounded on $L^{p(\cdot)}\left(\mathbb{R}^{n}\right)$. For the set $\mathscr{B}\left(\mathbb{R}^{n}\right)$, we refer the reader to $[19,41]$ for details. If $p(\cdot) \in \mathscr{B}\left(\mathbb{R}^{n}\right)$, we will use the following results.

Lemma 4 (see [22]). Let $p(\cdot) \in \mathscr{B}\left(\mathbb{R}^{n}\right)$. Then there exist $0<$ $\delta<1$ depending only on $p(\cdot)$ and $n$ such that for balls $B$ in $\mathbb{R}^{n}$ and all measurable subsets $S \subset B$

$$
\frac{\left\|\chi_{S}\right\|_{L^{p(\cdot)}\left(\mathbb{R}^{n}\right)}}{\left\|\chi_{B}\right\|_{L^{p(\cdot)}\left(\mathbb{R}^{n}\right)}} \leqslant C\left(\frac{|S|}{|B|}\right)^{\delta} .
$$

Lemma 5 (see $[22])$. Let $p(\cdot) \in \mathscr{B}\left(\mathbb{R}^{n}\right)$. Then there exists a positive constant $C>0$ such that for balls $B$ in $\mathbb{R}^{n}$,

$$
\frac{1}{|B|}\left\|\chi_{B}\right\|_{L^{p(\cdot)}\left(\mathbb{R}^{n}\right)}\left\|\chi_{B}\right\|_{L^{p^{\prime} \cdot(\cdot)\left(\mathbb{R}^{n}\right)}} \leqslant C,
$$

where and what follows $p^{\prime}(\cdot)$ is the conjugate exponent of $p(\cdot)$, which means $p^{\prime}(x)=p(x) /(p(x)-1)$.

Lemma 6 (see $[23,24])$. Suppose $p(\cdot) \in \mathscr{B}\left(\mathbb{R}^{n}\right)$, and then there exists a positive constant $C$ such that for each $b \in$ $\operatorname{BMO}\left(\mathbb{R}^{n}\right)$

$$
\begin{aligned}
C^{-1}\|b\|_{B M O\left(\mathbb{R}^{n}\right)} & \leq \sup _{\mathrm{Q}} \frac{\left\|\left(b-b_{\mathrm{Q}}\right) \chi_{\mathrm{Q}}\right\|_{L^{p(\cdot)}}}{\left\|\chi_{\mathrm{Q}}\right\|_{L^{p(\cdot)}}} \\
& \leq C\|b\|_{B M O\left(\mathbb{R}^{n}\right)} .
\end{aligned}
$$

Theorem 7. Let $0<\lambda(x)<n$ for $x \in \mathbb{R}^{n}$. Suppose $S$ is a linear or sublinear operator satisfying

$$
|S f(x)| \leqslant C \int_{\mathbb{R}^{n}} \frac{|f(y)|}{|x-y|^{n}} \mathrm{~d} y, \quad \text { for } x \notin \operatorname{supp} f .
$$

If $p(\cdot) \in \mathscr{B}\left(\mathbb{R}^{n}\right), \lambda^{+}<n \delta p^{-}$, where $\delta$ is as in Lemma 4 and the operator $S$ is bounded on $L^{p(\cdot)}\left(\mathbb{R}^{n}\right)$, then $S$ is also bounded on $L^{p(\cdot), \lambda(\cdot)}\left(\mathbb{R}^{n}\right)$. That means

$$
\|S f\|_{L^{p(\cdot), \lambda(\cdot)}} \leqslant C\|f\|_{L^{p(\cdot), \lambda(\cdot)}}
$$

where the constant $C$ is independent of $f$.

Proof. Let $f \in L^{p(\cdot), \lambda(\cdot)}$, pick any $t \in \mathbb{R}^{n}$, and write $f(x)=$ $f_{0}(x)+\sum_{i=1}^{\infty} f_{i}(x)$, where $f_{0}=\chi_{B(t, 2 r)} f, f_{i}=\chi_{B\left(t, 2^{i+1} r\right) \backslash B\left(t, 2^{i} r\right)} f$.

Firstly, we estimate $S f_{0}$ on $B(t, r)$. By the boundedness of $S$ on $L^{p(\cdot)}\left(\mathbb{R}^{n}\right)$, we have

$$
\begin{aligned}
\left\|S f_{0}\right\|_{L^{p(\cdot)}\left(\mathbb{R}^{n}\right)} & \leqslant C\left\|f_{0}\right\|_{L^{p(\cdot)}\left(\mathbb{R}^{n}\right)} \\
& \leqslant C v_{1} r^{\lambda(t) / p(t)}\|f\|_{L^{p(\cdot), \lambda(\cdot)}} .
\end{aligned}
$$

Thus,

$$
\begin{aligned}
\left\|S f_{0}\right\|_{L^{p(\cdot)}(B(t, r))} & \leqslant\left\|S f_{0}\right\|_{L^{p(\cdot)}\left(\mathbb{R}^{n}\right)} \\
& \leqslant C v_{1} r^{\lambda(t) / p(t)}\|f\|_{L^{p(\cdot), \lambda(\cdot)}} .
\end{aligned}
$$

Hence, we obtain

$$
\left\|S f_{0}\right\|_{L^{p(\cdot), \lambda(\cdot)}} \leqslant C\|f\|_{L^{p(\cdot), \lambda(\cdot)}} .
$$

It remains to estimate $S\left(\sum_{i=1}^{\infty} f_{i}\right)(x)$ on $B(t, r)$. By the size estimate of $S$, we have

$$
\left|S f_{i}(x)\right| \leqslant C\left(2^{i} r\right)^{-n} \int_{\mathbb{R}^{n}}\left|f_{i}(y)\right| \mathrm{d} y,
$$

for $x \in B(t, r)$

Thus, by Lemmas 4 and 5 and Hölder's inequality, we have

$$
\begin{aligned}
& \left\|S\left(\sum_{i=1}^{\infty} f_{i}\right)\right\|_{L^{p(\cdot)}(B(t, r))} \leqslant\left\|\sum_{i=1}^{\infty} S f_{i}\right\|_{L^{p(\cdot)}(B(t, r))} \\
& \quad \leqslant C \sum_{i=1}^{\infty}\left\|\left(2^{i} r\right)^{-n} \int_{\mathbb{R}^{n}}\left|f_{i}(y)\right| \mathrm{d} y\right\|_{L^{p(\cdot)}(B(t, r))} \\
& \quad \leqslant C \sum_{i=1}^{\infty}\left(2^{i} r\right)^{-n}\|1\|_{L^{p(\cdot)}(B(t, r))} \int_{B\left(t, 2^{i+1} r\right)}|f(y)| \mathrm{d} y
\end{aligned}
$$




$$
\begin{aligned}
& \leqslant C \sum_{i=1}^{\infty}\left(2^{i} r\right)^{-n}\|1\|_{L^{p(\cdot)}(B(t, r))}\|1\|_{L^{p^{(\cdot)}\left(B\left(t, 2^{i+1} r\right)\right)}} \\
& \cdot\|f\|_{L^{p(\cdot)}\left(B\left(t, 2^{i+1} r\right)\right)} \\
& \leqslant C \sum_{i=1}^{\infty}\left(2^{i} r\right)^{-n} \frac{\|1\|_{L^{p(\cdot)}(B(t, r))}}{\|1\|_{L^{p(\cdot)}\left(B\left(t, 2^{i+1} r\right)\right)}}\|1\|_{L^{p(\cdot)}\left(B\left(t, 2^{i+1} r\right)\right)} \\
& \times\|1\|_{L^{p^{\prime}(\cdot)}\left(B\left(t, 2^{i+1} r\right)\right)} v_{1}\left(2^{i+1} r\right)^{\lambda(t) / p(t)}\|f\|_{L^{p(\cdot), \lambda(\cdot)}} \\
& \leqslant C \sum_{i=1}^{\infty} 2^{-n \delta(i+1)} v_{1}\left(2^{i+1} r\right)^{\lambda(t) / p(t)}\|f\|_{L^{p(\cdot), \lambda(\cdot)}} .
\end{aligned}
$$

Thus,

$$
\begin{aligned}
\left\|S\left(\sum_{i=1}^{\infty} f_{i}\right)\right\|_{L^{p(\cdot), \lambda(\cdot)}} & \leqslant C \sum_{i=1}^{\infty} 2^{-(i+1)\left(n \delta-\lambda^{+} / p^{-}\right)}\|f\|_{L^{p(\cdot), \lambda(\cdot)}} \\
& \leqslant C\|f\|_{L^{p(\cdot), \lambda(\cdot)}} .
\end{aligned}
$$

This finishes the proof of Theorem 7.

Next we turn to the boundedness of commutators in variable Morrey spaces. Many authors have studied it; see [44], but they considered that it restricts the underlying space with finite measure.

Theorem 8. Suppose $S$ is a linear operator satisfying

$$
S f(x)=\int_{\mathbb{R}^{n}} \frac{\Omega(x-y)}{|x-y|^{n}} f(y) \mathrm{d} y, \quad \text { for } x \notin \operatorname{supp} f,
$$

where $\Omega$ is a bounded measurable function. Let $0<\lambda(x)<$ $n$, and $b \in B M O\left(\mathbb{R}^{n}\right)$ and $p(\cdot) \in \mathscr{B}\left(\mathbb{R}^{n}\right)$. If the commutator $[b, S]$ is bounded on $L^{p(\cdot)}\left(\mathbb{R}^{n}\right)$, then $[b, S]$ is also bounded on $L^{p(\cdot), \lambda(\cdot)}\left(\mathbb{R}^{n}\right)$.

Proof. For any $t \in \mathbb{R}^{n}$ and $r>0$, let $B=B(t, r)$ and write $f$ as in the proof of Theorem 7 . By the $L^{p(\cdot)}$-boundedness of $[b, S]$, we obtain

$$
\begin{aligned}
\left\|[b, S] f_{0}(x) \chi_{B}\right\|_{L^{p(\cdot)}} & \leq C\left\|f_{0}\right\|_{L^{p(\cdot)}} \\
& \leq C v_{1}(2 r)^{\lambda(\cdot) / p(x)}\|f\|_{L^{p(\cdot), \lambda(\cdot)}\left(\mathbb{R}^{n}\right)} .
\end{aligned}
$$

For $k>0$ and $x \in B$, we write

$$
\begin{aligned}
& \left|[b, S] f_{k}(x)\right| \leq \frac{C}{\left(2^{k} r\right)^{n}} \\
& \quad \cdot \int_{2^{k+1} B}\left|b(x)-b_{2 r}\right|\left|\Omega(x-y) f_{k}(y)\right| \mathrm{d} y+\frac{C}{\left(2^{k} r\right)^{n}} \\
& \quad \cdot \int_{2^{k+1} B}\left|b_{2 r}-b_{2^{k+1} r}\right|\left|\Omega(x-y) f_{k}(y)\right| \mathrm{d} y+\frac{C}{\left(2^{k} r\right)^{n}} \\
& \quad \cdot \int_{2^{k+1} B}\left|b(y)-b_{2^{k+1} r}\right|\left|\Omega(x-y) f_{k}(y)\right| \mathrm{d} y \\
& :=I_{1 k}(x)+I_{2 k}(x)+I_{3 k}(x),
\end{aligned}
$$

where and in what follows, for $k \geqslant 0, b_{2^{k+1} r}$ is defined by

$$
b_{2^{k+1} r}=\frac{1}{\left|B\left(t, 2^{k+1} r\right)\right|} \int_{B\left(t, 2^{k+1} r\right)} b(y) \mathrm{d} y .
$$

By the well-known fact that, for any $r>0$ and $k \in \mathbb{N}$, $\left|b_{2^{k+1} r}-b_{2 r}\right| \leq C_{n} k\|b\|_{*}$ (see [47, Proposition 7.1.5(i)]), we obtain

$$
\begin{aligned}
& I_{2 k}(x) \leq C(k+1)\|b\|_{*}\left(2^{k} r\right)^{-n} \int_{2^{k+1} B}\left|f_{k}(y)\right| \mathrm{d} y \\
& \quad \leq C(k+1)\left(2^{k} r\right)^{-n}\|b\|_{*}\left\|\chi_{2^{k+1} B}\right\|_{L^{p^{\prime}(\cdot)}}\|f\|_{L^{p(\cdot)}\left(2^{k+1} B\right)} .
\end{aligned}
$$

Hence, for $k>0$, using Lemmas 4 and 5 we have

$$
\begin{gathered}
\left\|I_{2 k} \chi_{B}\right\|_{L^{p(\cdot)}} \leq C(k+1)\left(2^{k} r\right)^{-n}\|b\|_{*}\left\|\chi_{B}\right\|_{L^{p(\cdot)}} \\
\cdot\left\|\chi_{2^{k+1} B}\right\|_{L^{p^{(\cdot)}}}\|f\|_{L^{p(\cdot)}\left(2^{k+1} B\right)} \leq C(k+1)\|b\|_{*} \\
\cdot 2^{-n \delta(k+1)} v_{1}\left(2^{k+1} r\right)^{\lambda(t) / p(t)}\|f\|_{L^{p(\cdot), \lambda(\cdot)}} \leq C(k+1) \\
\cdot\|b\|_{*} 2^{-(k+1)\left(n \delta-\lambda^{+} / p^{-}\right)} v_{1}(2 r)^{\lambda(t) / p(t)}\|f\|_{L^{p(\cdot), \lambda(\cdot)}} \cdot
\end{gathered}
$$

For $I_{3 k}(X)$, by Hölder's inequality and Lemma 6, we have $I_{3 k}(x)$

$$
\begin{aligned}
& \leq C \frac{1}{\left(2^{k} r\right)^{n}}\left\|\left(b-b_{2^{k+1} r}\right) \chi_{2^{k+1} B}\right\|_{L^{p^{(\cdot)}}}\|f\|_{L^{p(\cdot)}\left(2^{k+1} B\right)} \\
& \leq C \frac{1}{\left(2^{k} r\right)^{n}}\|b\|_{*}\left\|\chi_{2^{k+1} B}\right\|_{L^{\left.p^{(} \cdot\right)}}\|f\|_{L^{p(\cdot)}\left(2^{k+1} B\right)} .
\end{aligned}
$$

Therefore, as the argument as for $I_{2 k}$, we have

$\left\|I_{3 k} \chi_{B}\right\|_{L^{p(\cdot)}}$

$$
\leq C\|b\|_{*} 2^{-(k+1)\left(n \delta-\lambda^{+} / p^{-}\right)} v_{1}(2 r)^{\lambda(t) / p(t)}\|f\|_{L^{p(\cdot), \lambda(\cdot)}} .
$$

Finally, for $I_{1 k}(x)$ by Hölder's inequality, we have

$$
\begin{aligned}
& I_{1 k}(x) \\
& \quad \leq C\left|b(x)-b_{r}\right| \frac{1}{\left(2^{k} r\right)^{n}}\left\|\chi_{2^{k+1} B}\right\|_{L^{p^{\prime}(\cdot)}}\|f\|_{L^{p(\cdot)}\left(2^{k+1} B\right)} .
\end{aligned}
$$

Thus, as the argument as before, we obtain that

$\left\|I_{1 k} \chi_{B}\right\|_{L^{p(\cdot)}}$

$$
\begin{aligned}
& \leq C\|b\|_{*} \frac{1}{\left(2^{k} r\right)^{n}}\left\|\chi_{B}\right\|_{L^{p(\cdot)}}\left\|\chi_{2^{k+1} B}\right\|_{L^{p^{(}(\cdot)}}\|f\|_{L^{p^{(\cdot)}\left(2^{k+1} B\right)}} \\
& \leq C\|b\|_{*} 2^{-(k+1)\left(n \delta-\lambda^{+} / p^{-}\right)} v_{1}(2 r)^{\lambda(t) / p(t)}\|f\|_{L^{p(\cdot), \lambda(\cdot)}} .
\end{aligned}
$$

From (45), (47), and (49), we get

$$
\begin{aligned}
& \left\|[b, S] \sum_{k=1}^{\infty} f_{k} \chi_{B}\right\|_{L^{p(\cdot)}} \leq C \sum_{k=1}^{\infty}(k+1)\|b\|_{*} \\
& \cdot 2^{-(k+1)\left(n \delta-\lambda^{+} / p^{-}\right)} v_{1}(2 r)^{\lambda(t) / p(t)}\|f\|_{L^{p(\cdot), \lambda(\cdot)}} \leq C\|b\|_{*} \\
& \cdot v_{1}(2 r)^{\lambda(t) / p(t)}\|f\|_{L^{p(\cdot), \lambda(\cdot)}} .
\end{aligned}
$$


Hence,

$$
\|[b, S] f\|_{L^{p(\cdot), \lambda(\cdot)}\left(\mathbb{R}^{n}\right)} \leq C\|b\|_{*}\|f\|_{L^{p(\cdot), \lambda(\cdot)}\left(\mathbb{R}^{n}\right)} .
$$

This finishes the proof of Theorem 8 .

Corollary 9. Let $0<\lambda(x)<n$. Suppose that $\Omega$ is a bounded homogeneous function of degree 0 and satisfies conditions (2) and

$$
\int_{0}^{1} \frac{w(\delta)}{\delta} \mathrm{d} \delta<\infty
$$

where

$$
w(\delta)=\sup _{x^{\prime}, y^{\prime} \in S^{n-1},\left|x^{\prime}-y^{\prime}\right|<\delta}\left|\Omega\left(x^{\prime}\right)-\Omega\left(y^{\prime}\right)\right| .
$$

If $p(\cdot) \in \mathscr{B}\left(\mathbb{R}^{n}\right)$, then the Calderón-Zygmund singular integral operator $T$ defined by (1) and its commutator $[b, T]$ with $b \in$ $\operatorname{BMO}\left(\mathbb{R}^{n}\right)$ are both bounded on $L^{p(\cdot), \lambda(\cdot)}\left(\mathbb{R}^{n}\right)$.

Proof. Corollary 9 is the result of the following lemmas. Indeed, the boundedness of $T$ on $L^{p(\cdot), \lambda(\cdot)}\left(\mathbb{R}^{n}\right)$ is the direct result of Theorem 7, Lemmas 12 and 13 . For the commutator, if $w \in A_{p}, p \in(1, \infty)$, then by Corollary 9.2.6 in [47] there exists $\epsilon>0$ such that $w \in A_{p-\epsilon}$ and $p-\epsilon>1$. Then we choose $s=p /(p-\epsilon)<p$ in Lemma 11; for $w \in A_{p}$ by Lemma 10 we obtain

$$
\int_{\mathbb{R}^{n}}|[b, T] f(x)|^{p} w(x) \mathrm{d} x \leqslant C \int_{\mathbb{R}^{n}}|f(x)|^{p} w(x) \mathrm{d} x,
$$

for bounded functions $b$ and compactly supported functions $f$. Finally, using the similar argument for Theorem 7.5.6 in [47], we obtain that the last inequality holds for any $b \in$ $\operatorname{BMO}\left(\mathbb{R}^{n}\right)$. Thus, by Lemma 13 , we obtain that $[b, T]$ is bounded on $L^{p}(\cdot)\left(\mathbb{R}^{n}\right)$. Consequently, by Theorem $8,[b, T]$ is bounded on $L^{p(\cdot), \lambda(\cdot)}\left(\mathbb{R}^{n}\right)$.

We remark here that the boundedness in variable Lebesgue spaces $L^{p(\cdot)}\left(\mathbb{R}^{n}\right)$ of commutator $[b, T]$ has been proved in [44] by another method when $\Omega$ is an infinitely differentiable function on $S^{n-1}$.

Lemma 10 (see Lemma 2.1 in [48]). Let $1<p<\infty$ and $w \in$ $A_{p}$ (Muckenhoupt weight); then there exists a positive constant C such that

$$
\int_{\mathbb{R}^{n}} M f(x)^{p} w(x) \mathrm{d} x \leq C \int_{\mathbb{R}^{n}} M^{\#} f(x)^{p} w(x) \mathrm{d} x,
$$

for functions $f$ such that the left-hand side is finite.

Here we say $w \in A_{p}, 1<p<\infty$ if for every cube $Q$

$$
\begin{aligned}
& \left(\frac{1}{|Q|} \int_{Q} w(x) \mathrm{d} x\right)\left(\frac{1}{|Q|} \int_{Q} w(x)^{1-p^{\prime}} \mathrm{d} x\right)^{p-1} \leq C \\
& \quad<\infty
\end{aligned}
$$

For properties of $A_{p}$, we refer the reader to [47].
Lemma 11 (see Lemma 2.4.1 in [49]). Let $b \in B M O\left(\mathbb{R}^{n}\right)$ and $p \in(1, \infty)$. Then for any $s \in(1, p)$, there exists a constant $C$, independent of $b$ and $f$, such that

$$
\begin{aligned}
& M^{\#}[b, T](f)(x) \\
& \quad \leq C\|b\|_{*}\left[\left(M\left(|T f|^{s}\right)(x)\right)^{1 / s}+\left(M\left(|f|^{s}\right)(x)\right)^{1 / s}\right],
\end{aligned}
$$

$\forall x \in \mathbb{R}^{n}$.

Lemma 12 (see Theorem 2.1.6 in [49]). Suppose that $\Omega$ is a bounded homogeneous function of degree 0 and satisfies conditions (1) and (52). If $p \in(1, \infty)$ and $w \in A_{p}$, then there exists a constant $C$, independent of $f$, such that

$$
\int_{\mathbb{R}^{n}}|T f(x)|^{p} w(x) \mathrm{d} x \leq C \int_{\mathbb{R}^{n}}|f(x)|^{p} w(x) \mathrm{d} x .
$$

Lemma 13 (see Corollary 1.11 in [18]). Given that $\mathscr{F}$ denotes a family of ordered pairs of nonnegative, measurable functions $(f, g)$ on $\mathbb{R}^{n}$, assume that

$$
\begin{array}{r}
\int_{\mathbb{R}^{n}} f(x)^{p_{0}} w(x) \mathrm{d} x \leqslant C_{0} \int_{\mathbb{R}^{n}} g(x)^{p_{0}} w(x) \mathrm{d} x, \\
(f, g) \in \mathscr{F},
\end{array}
$$

holds for some $1<p_{0}<\infty$, for every $\omega \in A_{p_{0}}$ and for all $(f, g) \in \mathscr{F}$. Let $p(\cdot) \in \mathscr{B}\left(\mathbb{R}^{n}\right)$. Then

$$
\|f\|_{L^{p^{(\cdot)}}} \leqslant C\|g\|_{L^{p(\cdot)}} .
$$

\section{Compactness of Commutators}

Now we obtain sufficient conditions for the commutator $[b, T]$ to be a compact operator on $L^{p(\cdot), \lambda(\cdot)}\left(\mathbb{R}^{n}\right)$.

Theorem 14. Let $0<\lambda(x)<n$ for $x \in \mathbb{R}^{n}$ and $p(\cdot) \in \mathscr{B}\left(\mathbb{R}^{n}\right)$ such that $\lambda(\cdot) / p(\cdot)$ is a constant function. Suppose that $\Omega$ is a bounded homogeneous function of degree of 0 and satisfies (2) and for some $q \in\left(1, p^{-}\right)$

$$
\int_{0}^{1} \frac{\omega_{q}(\delta)}{\delta}(1+|\log \delta|) \mathrm{d} \delta<\infty
$$

where $\omega_{q}(\delta)$ denotes the integral modulus of continuity of order q of $\Omega$ defined by

$$
\omega_{q}(\delta)=\sup _{\|\rho\|<\delta}\left(\int_{S^{n-1}}\left|\Omega\left(\rho x^{\prime}\right)-\Omega\left(x^{\prime}\right)\right|^{q} \mathrm{~d} \sigma\left(x^{\prime}\right)\right)^{1 / q},
$$

and $\rho$ is a rotation in $\mathbb{R}^{n}$ and $\|\rho\|=\sup _{x^{\prime} \in S^{n-1}}\left|\rho x^{\prime}-x^{\prime}\right|$. If $b \in$ $V M O\left(\mathbb{R}^{n}\right)$ (the closure of the set of compactly supported infinite differential functions in $\left.B M O\left(\mathbb{R}^{n}\right)\right)$, then the commutator $[b, T]$ is a compact operator on $L^{p(\cdot), \lambda(\cdot)}\left(\mathbb{R}^{n}\right)$.

Lemma 15. Let $0<\lambda(\cdot)<n$. Suppose that $\Omega$ is a bounded homogeneous function of degree of 0 and satisfies (2) and (52). For $\eta>0$, let

$$
T_{\eta} f(x)=\int_{|x-y|>\eta} \frac{\Omega(x-y)}{|x-y|^{n}} f(y) \mathrm{d} y .
$$




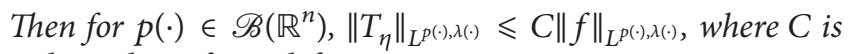
independent of $\eta$ and $f$.

Proof. Lemma 15 is a direct consequence of Theorem 7. In fact, by Theorem 2.1.8 in [49], given that $1<p<\infty$, if $\omega \in A_{p}$, then

$$
\int_{\Omega} T_{\eta} f(x)^{p} \omega(x) \mathrm{d} x \leqslant C_{0} \int_{\Omega} f(x)^{p} \omega(x) \mathrm{d} x
$$

holds uniformly in $\eta$. Using Lemma 13 , we can get that $T_{\eta}$ is bounded on $L^{p(\cdot)}\left(\mathbb{R}^{n}\right)$ uniformly in $\eta$. Now all conditions in Theorem 7 are fulfilled.

Lemma 16 (see Lemma 2.2 in [8]). Suppose that $0<\beta<n$, $\Omega$ satisfies (2), and $\Omega \in L^{q}\left(S^{n-1}\right)$, where $q>1$. Then there exist $C>0$ such that for an $R>0$ and $x \in \mathbb{R}^{n}$ with $|x|<R / 2$

$$
\begin{aligned}
& \left(\int_{R<|y|<2 R}\left|\frac{\Omega(y-x)}{|y-x|^{n-\beta}}-\frac{\Omega(y)}{|y|^{n-\beta}}\right|^{q} \mathrm{~d} y\right)^{1 / q} \\
& \quad \leqslant C R^{n / q-(n-\beta)}\left\{\frac{|x|}{R}+\int_{|x| / 2 R}^{|x| / R} \frac{w_{q}(\delta)}{\delta} \mathrm{d} \delta\right\} .
\end{aligned}
$$

Proof of Theorem 14. We will use the method in [8]. Let $\mathscr{F}$ be the unit ball in $L^{p(\cdot), \lambda(\cdot)}\left(\mathbb{R}^{n}\right)$. By density, we only need to prove that when $b \in C_{c}^{\infty}\left(\mathbb{R}^{n}\right)$, the set $\mathscr{G}=\{[b, T] f: f \in \mathscr{F}\}$ is a precompact in $L^{p(\cdot), \lambda(\cdot)}\left(\mathbb{R}^{n}\right)$. By Theorem 1 , it is sufficient to show that (11)-(13) hold uniformly in $\mathscr{G}$.

Notice that $b \in C_{c}^{\infty}\left(\mathbb{R}^{n}\right)$. Applying Corollary 9, we have

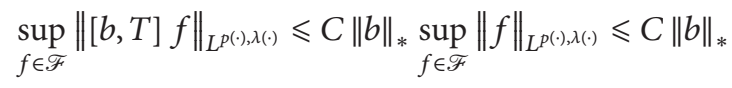

$$
<\infty \text {. }
$$

This shows that (11) holds.

Next we show that (13) holds. To do so, we suppose that $\beta>1$ taken so large that supp $b \subset\{x:|x| \leq \beta\}$. For any $0<\epsilon<1$, we take $\alpha>\beta$ such that $(\alpha-\beta)^{n(1-q)}<\epsilon^{q}$. Below we show that for every $t \in \mathbb{R}^{n}$ and $r>0, q>1$

$$
\frac{1}{v_{1} r^{\lambda(t) / p(t)}}\left\|[b, T] f \chi_{E_{\alpha}}\right\|_{L^{p(\cdot)} B(t, r)}<C \epsilon\|\Omega\|_{L^{q}\left(S^{n-1}\right)} .
$$

In fact, for any $x \in E_{\alpha}=\left\{x \in \mathbb{R}^{n}:|x|>\alpha\right\}$ and every $f \in \mathscr{F}$, by Hölder's inequality we have

$$
\begin{aligned}
& |[b, T] f(x)| \\
& \quad=\left|\int_{\mathbb{R}^{n}} \frac{\Omega(x-y)}{|x-y|^{n}}(b(x)-b(y)) f(y) \mathrm{d} y\right| \\
& \quad \leq C\|b\|_{\infty} \int_{|y|<\beta} \frac{|\Omega(x-y)|}{|x-y|^{n}}|f(y)| \mathrm{d} y \\
& \quad \leqslant C\left(\int_{|x-y| \leqslant \beta} \frac{|\Omega(y)|^{q}}{|y|^{n q}}|f(x-y)|^{q} \mathrm{~d} y\right)^{1 / q} .
\end{aligned}
$$

Then for every $t \in \mathbb{R}^{n}$ and $r>0$, by the Minkowski inequality and the choice of $\alpha$, we get

$$
\begin{aligned}
& \frac{1}{v_{1} r^{\lambda(t) / p(t)}}\left\|[b, T] f \chi_{E_{\alpha}}\right\|_{L^{p(\cdot)}(B(t, r))} \leqslant C \\
& \cdot \frac{1}{v_{1} r^{\lambda(t) / p(t)}} \|\left(\int_{|\cdot-y| \leq \beta} \frac{|\Omega(y)|^{q}}{|y|^{n q}}|f(\cdot-y)|^{q} \mathrm{~d} y\right)^{1 / q} \\
& \cdot \chi_{E_{\alpha}} \|_{L^{p(\cdot)}(B(t, r))} \leqslant C \\
& \cdot \frac{1}{v_{1} r^{\lambda(t) / p(t)}} \|\left(\int_{|-y| \leq \beta} \frac{|\Omega(y)|^{q}}{|y|^{n q}}|f(\cdot-y)|^{q} \mathrm{~d} y\right) \\
& \cdot \chi_{E_{\alpha}} \|_{L^{p(\cdot) / q(}(B(t, r))}^{1 / q} \leqslant C \frac{1}{r^{\lambda(t) / p(t)-\lambda(t-y) / p(t-y)}} \\
& \cdot \sup _{|x-y| \leqslant \beta} \frac{1}{v_{1} r^{\lambda(t-y) / p(t-y)}}\|f\|_{L^{p(\cdot)} B(t-y, r)} \\
& \cdot\left\{\int_{|y|>\alpha-\beta} \frac{|\Omega(y)|^{q}}{|y|^{n q}} \mathrm{~d} y\right)^{1 / q} \leqslant C \epsilon\|\Omega\|_{L^{q}\left(S^{n-1}\right)} \\
& \cdot\|f\|_{L^{p(\cdot), \lambda(\cdot)}} \cdot
\end{aligned}
$$

Thus, we get (67), which shows that (13) holds for $[b, T]$ in $\mathscr{G}$ uniformly.

Finally, we show that the translation continuity condition (12) holds for the commutator $[b, T]$ in $\mathscr{G}$ uniformly. We need to prove that, for any $0<\epsilon<1 / 2$, if $|z|$ is sufficiently small depending only on $\epsilon$, then for every $f \in \mathscr{F}$

$$
\|[b, T] f(\cdot)-[b, T] f(\cdot+z)\|_{L^{p(\cdot), \lambda(\cdot)}} \leqslant C \epsilon .
$$

Now for $z \in \mathbb{R}^{n}$, we write

$$
\begin{aligned}
& {[b, T] f(x+z)-[b, T] f(x)} \\
& \quad=\int_{|x-y|>e^{1 / \epsilon}|z|} \frac{\Omega(x-y)}{|x-y|^{n}}[b(x+z)-b(x)] \\
& \cdot f(y) \mathrm{d} y \\
& +\int_{|x-y|>e^{1 / \epsilon}|z|}\left(\frac{\Omega(x-y)}{|x-y|^{n}}-\frac{\Omega(x+z-y)}{|x+z-y|^{n}}\right) \\
& \cdot[b(y)-b(x+z)] f(y) \mathrm{d} y \\
& +\int_{|x-y| \leqslant e^{1 / \epsilon}|z|} \frac{\Omega(x-y)}{|x-y|^{n}}[b(y)-b(x)] f(y) \mathrm{d} y \\
& -\int_{|x-y| \leqslant e^{1 / \epsilon}|z|} \frac{\Omega(x+z-y)}{|x-y|^{n}}[b(y)-b(x+z)] \\
& \cdot f(y) \mathrm{d} y:=J_{1}+J_{2}+J_{3}-J_{4} .
\end{aligned}
$$

Since $b \in C_{c}^{\infty}\left(\mathbb{R}^{n}\right)$, we have $|b(x)-b(x+z)| \leqslant C\|\nabla b\|_{\infty}|z|$. Since $\Omega \in L^{q}\left(S^{n-1}\right)$ and applying Lemma 15 , we get

$$
\left\|J_{1}\right\|_{L^{p^{(\cdot), \lambda(\cdot)}}} \leqslant C|z|\|f\|_{L^{p(\cdot), \lambda(\cdot)}}<C|z| .
$$


As for $J_{2}$, for every $t \in \mathbb{R}^{n}$ and $r>0$, using Lemma 16 and the Minkowski inequality, we get

$$
\begin{aligned}
& \frac{1}{v_{1} r^{\lambda(t) / p(t)}}\left\|J_{2}\right\|_{L^{p(\cdot)}(B(t, r))} \leqslant 2\|b\|_{\infty} \frac{1}{v_{1} r^{\lambda(t) / p(t)}} \\
& \sup _{|x-y|>e^{1 / \epsilon}|z|}\|f\|_{L^{p(\cdot)}(B(t-y, r))} \int_{|y|>e^{1 / \epsilon}|z|} \mid \frac{\Omega(y)}{|y|^{n}} \\
& -\frac{\Omega(y+z)}{|y+z|^{n} \mid} \mid \mathrm{d} y \leqslant C \\
& \cdot \frac{1}{r^{\lambda(t) / p(t)-\lambda(t-y) / p(t-y)}}\|f\|_{L^{p(\cdot), \lambda(\cdot)}} \sum_{k=0}^{\infty}\left\{\frac{|z|}{2^{k} e^{1 / \epsilon}|z|}\right. \\
& \left.+\int_{|z| / 2^{k+1} e^{1 / \epsilon}|z|}^{|z| / 2^{k} e^{1 / \epsilon}|z|} \frac{\omega(\delta)}{\delta} \mathrm{d} \delta\right\} \leqslant C\|f\|_{L^{p(\cdot), \lambda(\cdot)}} \sum_{k=0}^{\infty}\left\{\frac{1}{2^{k} e^{1 / \epsilon}}\right. \\
& \left.+\frac{1}{1+k+1 / \epsilon} \int_{|z| / 2^{k+1} e^{1 / \epsilon}|z|}^{|z| / 2^{k} e^{1 / \epsilon}|z|} \frac{\omega(\delta)}{\delta}(1+|\log \delta|) \mathrm{d} \delta\right\} \\
& \leqslant C\left(e^{-1 / \epsilon}+\epsilon\right)\|f\|_{L^{p(\cdot), \lambda(\cdot)}} \text {. }
\end{aligned}
$$

Thus, we have

$$
\left\|J_{2}\right\|_{L^{p(\cdot), \lambda(\cdot)}} \leqslant C \epsilon .
$$

Regarding $J_{3}$, we have $|b(x)-b(y)| \leqslant C\|\nabla b\|_{\infty}|x-y|$ by $b \in C_{c}^{\infty}\left(\mathbb{R}^{n}\right)$. Thus,

$$
J_{3} \leqslant C \int_{|x-y| \leqslant e^{1 / \epsilon}|z|}|\Omega(x-y)||x-y|^{-n+1}|f(y)| \mathrm{d} y .
$$

By the Minkowski inequality, for every $t \in \mathbb{R}^{n}$ and $r>0$, we have

$$
\begin{aligned}
& \frac{1}{v_{1} r^{\lambda(t) / p(t)}}\left\|J_{3}\right\|_{L^{p^{(\cdot)}(B(t, r))}} \leqslant C \frac{1}{v_{1} r^{\lambda(t) / p(t)}} \\
& \cdot \sup _{|x-y| \leqslant e^{1 / \epsilon}|z|}\|f\|_{L^{p(\cdot)}(B(t-y, r))} \\
& \cdot \int_{|y| \leqslant e^{1 / \epsilon}|z|}|\Omega(y)||y|^{-n+1} \mathrm{~d} y \leqslant C \\
& \cdot \frac{1}{r^{\lambda(t) / p(t)-\lambda(t-y) / p(t-y)}} e^{1 / \epsilon}|z|\|f\|_{L^{p(\cdot), \lambda(\cdot)} \leqslant C e^{1 / \epsilon}|z|} \\
& \cdot\|f\|_{L^{p(\cdot), \lambda(\cdot)}} .
\end{aligned}
$$

Thus,

$$
\left\|J_{3}\right\|_{L^{p(\cdot), \lambda(\cdot)}} \leqslant C e^{1 / \epsilon}|z| .
$$

Finally, by $|b(x+z)-b(y)| \leqslant C\|\nabla b\|_{\infty}|x+z-y|$, we have

$$
J_{4} \leqslant C \int_{|x-y| \leqslant e^{1 / \epsilon}|z|}|\Omega(x+z-y)||x+z-y|^{-n+1} \mathrm{~d} y .
$$

Using the same argument for $J_{3}$, it is easy to check that

$$
\left\|J_{4}\right\|_{L^{p(\cdot), \lambda(\cdot)}} \leqslant C\left(e^{1 / \epsilon}|z|+|z|\right) \text {. }
$$

From (72), (74), (77), and (79), and taking $|z|$ to be sufficiently small, we can get

$$
\begin{aligned}
& \|[b, T] f(\cdot)-[b, T] f(\cdot+z)\|_{L^{p(\cdot), \lambda(\cdot)}} \\
& \quad \leqslant\left\|J_{1}\right\|_{L^{p(\cdot), \lambda(\cdot)}}+\left\|J_{2}\right\|_{L^{p(\cdot), \lambda(\cdot)}}+\left\|J_{3}\right\|_{L^{p(\cdot), \lambda(\cdot)}}+\left\|J_{4}\right\|_{L^{p(\cdot), \lambda(\cdot)}} \\
& \quad \leqslant C \epsilon .
\end{aligned}
$$

Therefore, we show that the translation continuity (12) holds for the commuator $[b, T]$ in $\mathscr{G}$ uniformly and this completes the proof of Theorem 14 .

We remark that in Theorem 14 the condition $b \in$ $\operatorname{VMO}\left(\mathbb{R}^{n}\right)$ is necessary by Theorem 1.2 in [8].

\section{Conflicts of Interest}

The authors declare that they have no conflicts of interest.

\section{Acknowledgments}

The first author was supported by the TianYuan Special Funds of the National Natural Science Foundation of China (Grant no. 11426221) and the High Level Introduction of Talent Research Start-Up Fund by Central South University of Forestry and Technology (Grant no. 1040212). The second author is supported by the National Natural Science Foundation of China (Grant no. 11361020).

\section{References}

[1] R. R. Coifman, R. Rochberg, and G. Weiss, "Factorization theorems for Hardy spaces in several variables," Annals of Mathematics, vol. 103, no. 3, pp. 611-635, 1976.

[2] A. Uchiyama, "On the compactness of operators of Hankel type," The Tohoku Mathematical Journal. Second Series, vol. 30, no. 1, pp. 163-171, 1978.

[3] S. Janson, "Mean oscillation and commutators of singular integral operators," Arkiv för Matematik, vol. 16, no. 2, pp. 263270, 1978.

[4] R. Coifman, P. Lions, Y. Meyer, and S. Semmes, "Compensated compactness and Hardy spaces," Journal de Mathématiques Pures et Appliquées, vol. 72, pp. 247-286, 1993.

[5] F. Beatrous and S.-Y. Li, "On the boundedness and compactness of operators of Hankel type," Journal of Functional Analysis, vol. 111, no. 2, pp. 350-379, 1993.

[6] Y. Chen and Y. Ding, "Compactness of the commutators of parabolic singular integrals," Science China. Mathematics, vol. 53, no. 10, pp. 2633-2648, 2010.

[7] Y. Chen, Y. Ding, and X. Wang, "Compactness of commutators of Riesz potential on Morrey spaces," Potential Analysis, vol. 30, no. 4, pp. 301-313, 2009.

[8] Y. Chen, Y. Ding, and X. Wang, "Compactness of commutators for singular integrals on Morrey spaces," Canadian Journal of Mathematics. Journal Canadien de Mathématiques, vol. 64, no. 2, pp. 257-281, 2012.

[9] C. Morrey, "On the solutions of quasi-linear elliptic partial differential equations," Transactions of the American Mathematical Society, vol. 43, no. 1, pp. 126-166, 1938. 
[10] T. Kato, "Strong solutions of the Navier-Stokes equation in Morrey spaces," Boletim da Sociedade Brasileira de Matemática, vol. 22, no. 2, pp. 127-155, 1992.

[11] Z. Shen, "The periodic schrödinger operators with potentials in the morrey class," Journal of Functional Analysis, vol. 193, no. 2, pp. 314-345, 2002.

[12] D. R. Adams and J. Xiao, "Nonlinear potential analysis on Morrey spaces and their capacities," Indiana University Mathematics Journal, vol. 53, no. 6, pp. 1629-1663, 2004.

[13] D. R. Adams and J. Xiao, "Regularity of Morrey commutators," Transactions of the American Mathematical Society, vol. 364, no. 9, pp. 4801-4818, 2012.

[14] D. R. Adams and J. Xiao, "Restrictions of Riesz-Morrey potentials," Arkiv för Matematik, vol. 54, no. 2, pp. 201-231, 2016.

[15] A. Almeida, J. Hasanov, and S. Samko, "Maximal and potential operators in variable exponent Morrey spaces," Georgian Mathematical Journal, vol. 15, no. 2, pp. 195-208, 2008.

[16] A. Almeida and D. Drihem, "Maximal, potential and singular type operators on Herz spaces with variable exponents," Journal of Mathematical Analysis and Applications, vol. 394, no. 2, pp. 781-795, 2012.

[17] A. Almeida and P. Hästö, "Besov spaces with variable smoothness and integrability," Journal of Functional Analysis, vol. 258, no. 5, pp. 1628-1655, 2010.

[18] D. Cruz-Uribe, A. Fiorenza, J. M. Martell, and C. Pérez, "The boundedness of classical operators on variable Lp spaces," Annales Academice Scientiarum Fennica Mathematica, vol. 31, no. 1, pp. 239-264, 2006.

[19] L. Diening, P. Harjulehto, P. Hästö, and M. Ruzicka, Lebesgue and Sobolev Spaces with Variable Exponents, vol. 2017 of Lecture Notes in Mathematics, Springer, Berlin, Germany, 2011.

[20] B. Dong and J. Xu, "Herz-Morrey type Besov and TriebelLizorkin spaces with variable exponents," Banach Journal of Mathematical Analysis, vol. 9, no. 1, pp. 75-101, 2015.

[21] J. Fu and J. Xu, "Characterizations of Morrey type Besov and Triebel-Lizorkin spaces with variable exponents," Journal of Mathematical Analysis and Applications, vol. 381, no. 1, pp. 280298, 2011.

[22] M. Izuki, "Boundedness of sublinear operators on Herz spaces with variable exponent and application to wavelet characterization," Analysis Mathematica, vol. 36, no. 1, pp. 33-50, 2010.

[23] M. Izuki, "Boundedness of commutators on Herz spaces with variable exponent," Rendiconti del Circolo Matematico di Palermo. Second Series, vol. 59, no. 2, pp. 199-213, 2010.

[24] M. Izuki and Y. Sawano, "Variable Lebesgue norm estimates for BMO functions," Czechoslovak Mathematical Journal, vol. 62(137), no. 3, pp. 717-727, 2012.

[25] H. Kempka, "2-microlocal Besov and Triebel-Lizorkin spaces of variable integrability," Revista Matemática Complutense, vol. 22, no. 1, pp. 227-251, 2009.

[26] H. Kempka, "Atomic, molecular and wavelet decomposition of generalized 2-microlocal Besov spaces," Journal of Function Spaces and Applications, vol. 8, no. 2, pp. 129-165, 2010.

[27] H. Kempka and J. Vybíral, "Lorentz spaces with variable exponents," Mathematische Nachrichten, vol. 287, no. 8-9, pp. 938-954, 2014.

[28] O. Kovácik and J. Rákosník, "On spaces Lp(x) and Wk,p(x)," Czechoslovak Mathematical Journal, vol. 41, no. 116, pp. 592-618, 1991.

[29] E. Nakai and Y. Sawano, "Hardy spaces with variable exponents and generalized Campanato spaces," Journal of Functional Analysis, vol. 262, no. 9, pp. 3665-3748, 2012.
[30] T. Noi, "Fourier multiplier theorems for Besov and TriebelLizorkin spaces with variable exponents," Mathematical Inequalities \& Applications, vol. 17, no. 1, pp. 49-74, 2014.

[31] T. Noi, "Trace and extension operators for Besov spaces and Triebel-Lizorkin spaces with variable exponents," Revista Matemática Complutense, vol. 29, no. 2, pp. 341-404, 2016.

[32] T. Noi and Y. Sawano, "Complex interpolation of Besov spaces and Triebel-Lizorkin spaces with variable exponents," Journal of Mathematical Analysis and Applications, vol. 387, no. 2, pp. 676690, 2012.

[33] T. Noi and Y. Sawano, "Weighted variable modulation spaces," Scientiae Mathematicae Japonicae, vol. 78, pp. 29-43, 2015.

[34] H. Rafeiro and S. Samko, "Variable exponent Campanato spaces," Journal of Mathematical Sciences (New York), vol. 172, no. 1, pp. 143-164, 2011.

[35] S. Samko, "Variable exponent Herz spaces," Mediterranean Journal of Mathematics, vol. 10, no. 4, pp. 2007-2025, 2013.

[36] C. Shi and J. Xu, "Herz type Besov and Triebel-Lizorkin spaces with variable exponent," Frontiers of Mathematics in China, vol. 8, no. 4, pp. 907-921, 2013.

[37] H. Wang and Z. Liu, "The Herz-type HARdy spaces with variable exponent and their applications," Taiwanese Journal of Mathematics, vol. 16, no. 4, pp. 1363-1389, 2012.

[38] J. Xu, "Variable Besov and Triebel-Lizorkin spaces," Annales Academice Scientiarum Fennice Mathematica, vol. 33, no. 2, pp. 511-522, 2008.

[39] J. S. Xu, "The relation between variable Bessel potential spaces and Triebel-Lizorkin spaces," Integral Transforms and Special Functions. An International Journal, vol. 19, no. 7-8, pp. 599-605, 2008.

[40] J. Xu and X. Yang, "Herz-Morrey-Hardy spaces with variable exponents and their applications," Journal of Function Spaces, vol. 2015, Article ID 160635, 19 pages, 2015.

[41] D. Cruz-Uribe and A. Fiorenza, Variable Lebesgue Spaces, Birkhäuser, Basel, Switzerland, 2013.

[42] V. S. Guliyev, J. J. Hasanov, and S. G. Samko, "Boundedness of maximal, potential type, and singular integral operators in the generalized variable exponent Morrey type spaces," Journal of Mathematical Sciences (New York), vol. 170, no. 4, pp. 423-443, 2010.

[43] A. Y. Karlovich and A. K. Lerner, "Commutators of singular integrals on generalized $L^{p}$ spaces with variable exponent," Publicacions Matemátiques, vol. 49, no. 1, pp. 111-125, 2005.

[44] V. Kokilashvili and A. Meskhi, "Boundedness of maximal and singular operators in Morrey spaces with variable exponent," Armenian Journal of Mathematics, vol. 1, no. 1, pp. 18-28, 2008.

[45] H. Rafeiro, "Kolmogorov compactness criterion in variable exponent Lebesgue spaces," Proceedings of A. Razmadze Mathematical Institute, vol. 150, pp. 105-113, 2009.

[46] R. A. Adams and J. Fournier, Sobolev Spaces, Academic Press, Heidelberg, Germany, 2nd edition, 2006.

[47] L. Grafakos, Modern Fourier Analysis, vol. 250 of Graduate Texts in Mathematics, Springer, New York, NY, USA, 2009.

[48] C. Pérez and R. Trujillo-González, "Sharp weighted estimates for multilinear commutators," Journal of the London Mathematical Society. Second Series, vol. 65, no. 3, pp. 672-692, 2002.

[49] S. Lu, Y. Ding, and D. Yan, Singular Integrals and Related Topics, World Scientific Publishing, Singapore, 2007. 


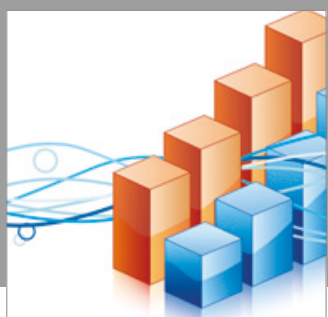

Advances in

Operations Research

vatersals

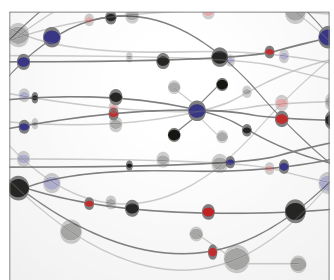

\section{The Scientific} World Journal
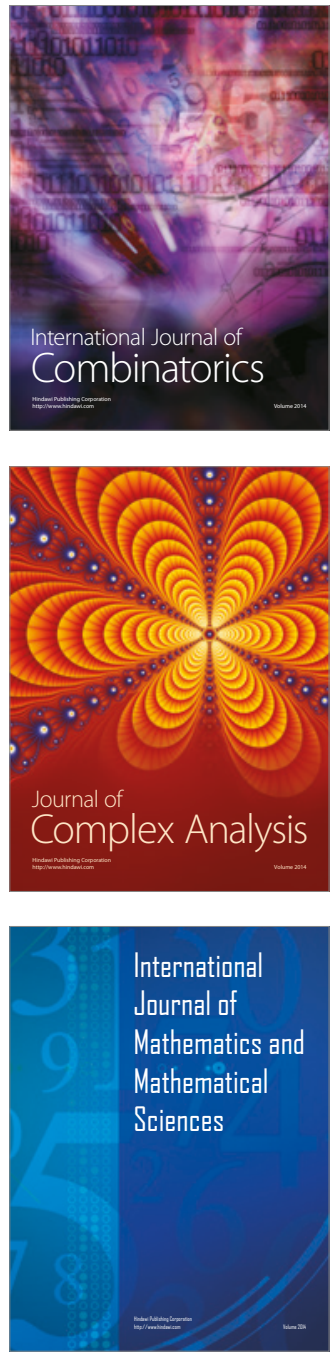
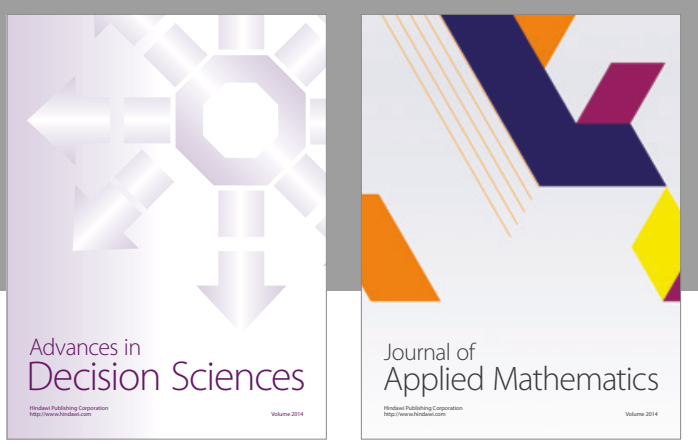

Algebra

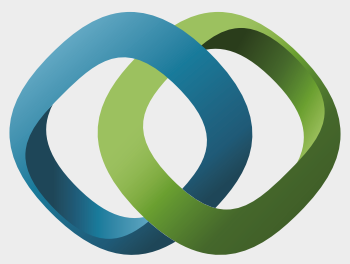

\section{Hindawi}

Submit your manuscripts at

https://www.hindawi.com
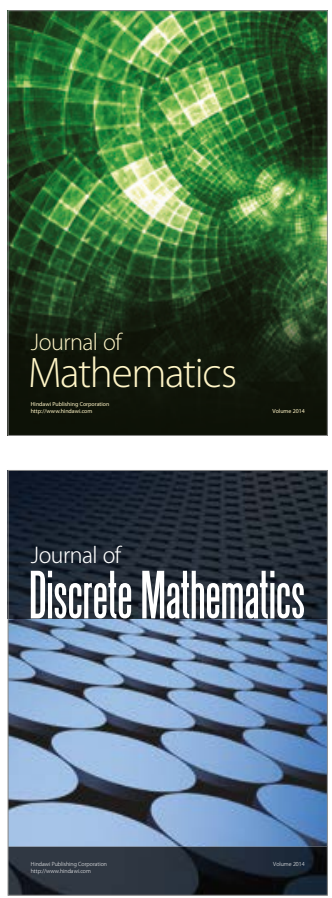

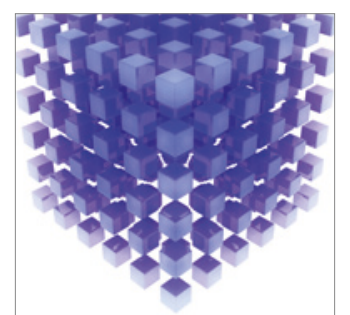

Mathematical Problems in Engineering
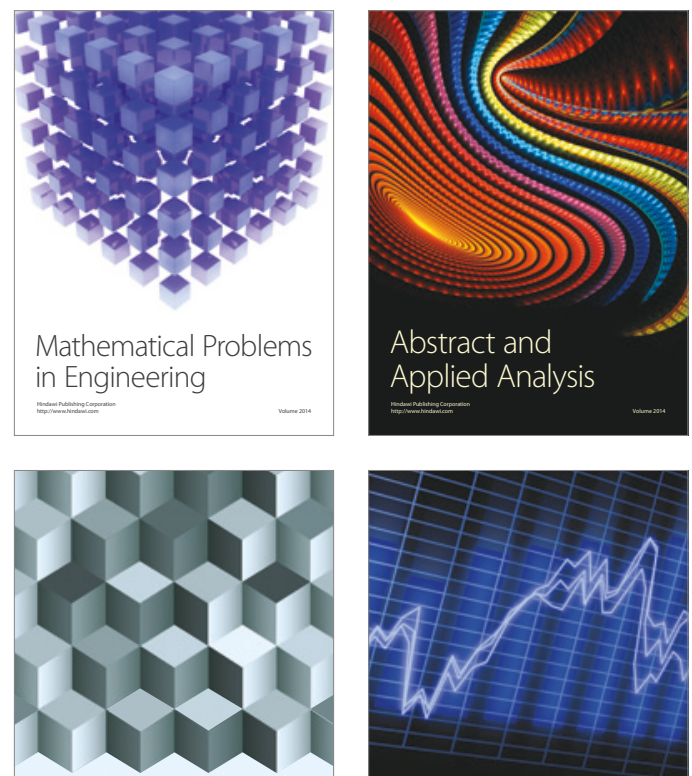

Journal of

Function Spaces

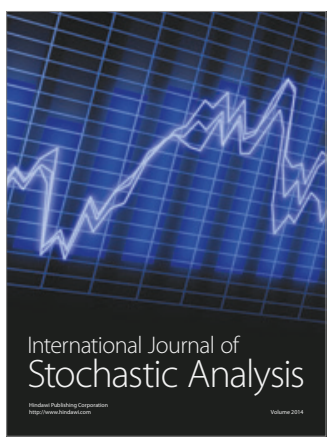

Probability and Statistics
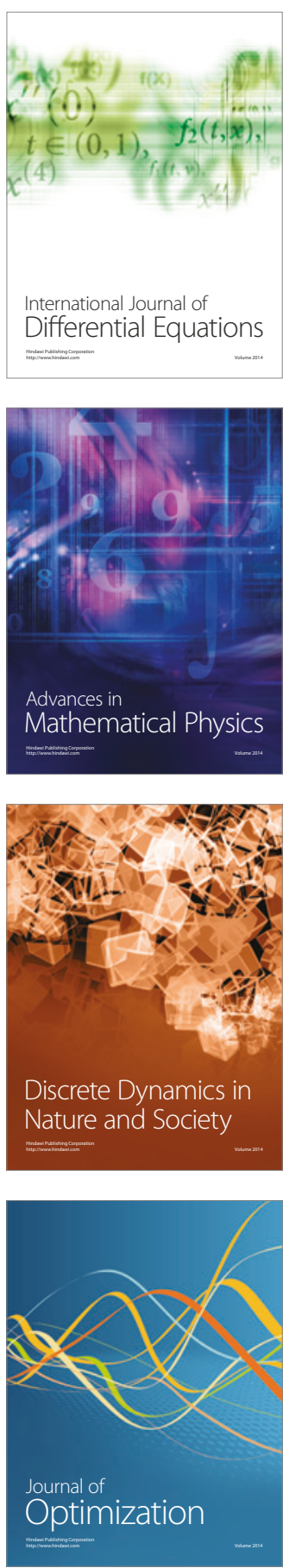